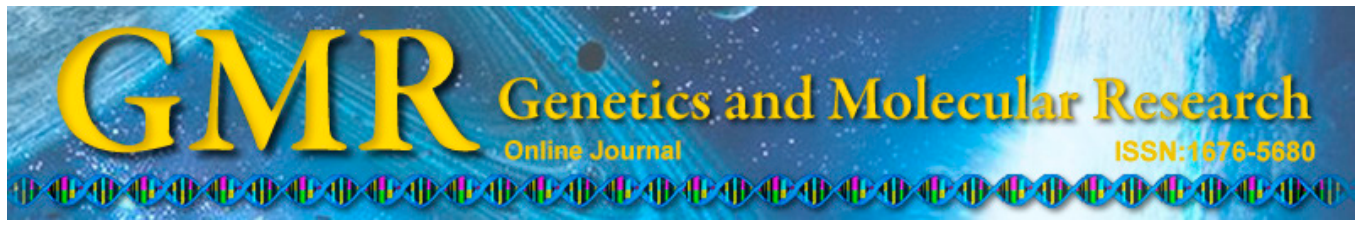

\title{
Lack of association between the $h O G G 1$ gene Ser326Cys polymorphism and gastric cancer risk: evidence from a case-control study and a meta-analysis
}

\author{
W.G. Hu ${ }^{1 *}$, R.J. Pan ${ }^{1 *}$, W. Cai ${ }^{1}$, Z.T. Wang ${ }^{2}$ and Z.G. Zhu ${ }^{1}$ \\ ${ }^{1}$ Department of General Surgery, Ruijin Hospital, \\ Shanghai Jiaotong University School of Medicine, Shanghai, China \\ ${ }^{2}$ Department of Gastroenterology, Ruijin Hospital, \\ Shanghai Jiaotong University School of Medicine, Shanghai, China \\ *These authors contributed equally to this study. \\ Corresponding author: Z.T. Wang \\ E-mail: dake_wang@126.com
}

Genet. Mol. Res. 14 (4): 14670-14679 (2015)

Received December 1, 2014

Accepted May 26, 2015

Published November 18, 2015

DOI http://dx.doi.org/10.4238/2015.November.18.31

\begin{abstract}
The association between the human 8-oxoguanine glycosylase 1 (hOGG1) gene Ser326Cys polymorphism (rs1052133) and gastric cancer has been widely evaluated, yet a definitive answer to whether this association exists is lacking. We first conducted a case-control study to assess this association in a large Han Chinese population, and then performed a meta-analysis to further address this issue. This case-control study involved 448 patients clinically diagnosed with gastric cancer and 372 cancer-free control individuals from China. Genotyping was conducted using the polymerase chain reaction-ligase detection reaction method. Meta-analysis was performed by the STATA software. Data and study quality were assessed in duplicate. Our case-control association study indicated that there were no significant differences in the genotype and allele distributions of the Ser326Cys
\end{abstract}


polymorphism between gastric cancer patients and controls $(\mathrm{P}=0.8026$ for genotype, and $\mathrm{P}=0.5857$ for allele), consistent with the results of the subsequent meta-analysis involving 2745 patients and 4588 controls under both allelic [odds ratio $(\mathrm{OR})=1.02 ; 95 \%$ confidence interval $(\mathrm{CI})$ $=0.91-1.14 ; \mathrm{P}=0.739]$ and dominant $(\mathrm{OR}=0.97 ; 95 \% \mathrm{CI}=0.78-1.21$; $\mathrm{P}=0.803$ ) models. Further subgroup analyses by ethnicity, source of controls, and sample size also did not detect any positive associations in this meta-analysis. Overall, our study in the Han Chinese population, along with the meta-analysis, failed to confirm the association of the $h O G G 1$ gene Ser326Cys polymorphism with gastric cancer risk, even across different ethnic populations.

Key words: Gastric cancer; $h O G G 1$ gene; Polymorphism; Risk association study; Meta-analysis

\section{INTRODUCTION}

Gastric cancer is one of the most common cancers worldwide and a leading cause of cancer-related mortality. Although the incidence of gastric cancer has gradually decreased in many Western countries, the highest incidence and mortality remains in East Asian countries (Long et al., 2010). The etiology of gastric carcinogenesis is still not fully understood. It is generally accepted that development of gastric cancer is a complex, multistep and multifactorial process involving a variety of risk factors. To date, a number of environmental risk factors including smoking, drinking, micronutrient deficiency, and Helicobacter pylori infection have been identified. However, not all people exposed to the above factors will develop gastric cancer, suggesting genetic involvement in gastric carcinogenesis.

Recently, it has been widely accepted that DNA damage plays an important role in the process of tumor generation and development. The base-excision repair pathway, which is composed of many DNA repair genes and has the function of removing DNA damage caused by ionizing radiation and reactive oxidative species, has attracted widespread attention as a potential mediator of tumorigenesis. The human 8-oxoguanine glycosylase 1 ( $h O G G 1$ ) gene, located on chromosome $3 \mathrm{p} 26$, is one component of the base excision response pathway and plays an important role the repair of damaged DNA. $h O G G 1$ encodes a DNA glycosylase enzyme that actively removes 8-hydroy-2-deoxyguanine, which is highly mutagenic and a major form of oxidative DNA damage (Collins and Gaivão, 2007). Therefore, the hOGG1 gene has been regarded as a logical candidate for involvement in the underlying cause of cancer. Meanwhile, a frequent polymorphism in the hOGG1 gene, rs1052133 (also known as the Ser326Cys polymorphism), results in the substitution of serine by cysteine at amino acid 326 of the hOGG1 protein and has been associated with an altered risk for various types of cancers in certain populations (Yuan et al., 2010; Wang et al., 2013). Functional studies have revealed that the hOGG1-Cys326 protein variant appears to have normal enzymatic activity, but maintains greater sensitivity to oxidation than does the serine variant (Kohno et al., 1998). Recently, the hOGG1 Ser326Cys polymorphism has been widely evaluated in association with gastric cancer across various ethnicities, yet with conflicting results, possibly due to insufficient sample sizes, genetic backgrounds, and selection of study populations. 
In this study, we first decided to assess the association of the hOGG1 gene Ser326Cys polymorphism gastric cancer risk in a large Han Chinese population. Then, given the accumulating data and to shed some light on recent conflicting or inconclusive claims, we sought to conduct a comprehensive meta-analysis of this association from both English and Chinese published literature.

\section{MATERIAL AND METHODS}

\section{Study population}

This was a hospital-based case-control study with a total of 820 subjects consecutively recruited from Shanghai Ruijin Hospital, China from May 2009 to December 2012 as previous described (Hu et al., 2014). The study population included 448 unrelated patients with histopathologically confirmed gastric cancer and 372 cancer-free controls; all subjects were local residents of Han descent. This study was approved by the Ethics Committee of Shanghai Jiaotong University School of Medicine, and was conducted according to the Declaration of Helsinki Principles. All subjects signed a written informed consent.

\section{Genotyping}

Blood samples $(1 \mathrm{~mL})$ were collected, and genomic DNA was extracted from white blood cells using the TIANamp Blood DNA Kit [Tiangen Biotect (Beijing) Co., Ltd., Beijing, China]. Genotyping was conducted using the polymerase chain reaction-ligase detection reaction (PCR-LDR) method using an ABI 9600 system (Applied Biosystems, Foster City, CA, USA) (Wang et al., 2014). Cycling parameters were as follows: $94^{\circ} \mathrm{C}$ for $2 \mathrm{~min} ; 35$ cycles of $94^{\circ} \mathrm{C}$ for $15 \mathrm{~s} ; 60^{\circ} \mathrm{C}$ for $15 \mathrm{~s}$; and $72^{\circ} \mathrm{C}$ for $30 \mathrm{~s}$; and a final extension step at $72^{\circ} \mathrm{C}$ for $5 \mathrm{~min}$. Two specific probes to discriminate the specific bases and one common probe were synthesized (Ramaniuk et al., 2014). The common probe was labeled at the 3 ' end with 6-carboxy-fluorescein and phosphorylated at the $5^{\prime}$ end. The reaction conditions for the LDR were: $94^{\circ} \mathrm{C}$ for $2 \mathrm{~min}$, 20 cycles of $94^{\circ} \mathrm{C}$ for $30 \mathrm{~s}$, and $60^{\circ} \mathrm{C}$ for $3 \mathrm{~min}$. After the reaction was completed, an aliquot $(1$ $\mu \mathrm{L}) \mathrm{LDR}$ products were mixed with $1 \mu \mathrm{L}$ ROX passive reference dye and $1 \mu \mathrm{L}$ loading buffer, denatured at $95^{\circ} \mathrm{C}$ for $3 \mathrm{~min}$, and chilled rapidly in ice water. The fluorescent products of LDR were differentiated using an ABI sequencer 377 (Applied Biosystems).

\section{Statistical analysis}

Comparisons between patients with gastric cancer and controls were conducted by unpaired $t$-test for continuous variables and by $\chi^{2}$ test for categorical variables. To avoid gross genotyping error, the Ser326Cys polymorphism was checked for consistency with Hardy-Weinberg equilibrium by the $\chi^{2}$ test. Genotypes were compared by conditional logistic regression analysis under assumptions of additive, dominant, and recessive models of inheritance, respectively. Statistical significance was accepted as $\mathrm{P}<0.05$.

\section{Meta-analysis}

Studies with the potential to be included in the meta-analysis were identified by 
searches of the PubMed, EMBASE, ISI Web of Knowledge, and China WANFANG (www. wanfangdata.com.cn) databases for relevant articles published as of March, 2014. Key subjects searched in Boolean combinations were "human 8-oxoguanine glycosylase or OGG1 or hOGG1" and "gastric cancer OR gastric carcinoma" and "polymorphism or allele or genotype or variant or variation". Search results were restricted to human populations and articles were written in English or Chinese. If more than one geographic or ethnically heterogeneous group was reported in a single article, each group was treated separately.

Studies were qualified for inclusion in the meta-analysis if they met the following criteria: i) based on a retrospective or nested case-control design; ii) adopted a validated genotyping method; and iii) provided genotype counts of the hOGG1 gene Ser326Cys polymorphism between patients with gastric cancer and controls.

In the meta-analysis, we assessed the association of the $h O G G 1$ gene $326 \mathrm{Ser}$ allele with gastric cancer relative to the 326Cys allele (allelic model), as well as the homozygous contrast, the dominant model, and the recessive model, respectively. Unadjusted odds ratios (ORs) and 95\% confidence intervals (CIs) were used to compare allele or genotype differences between patients and controls. The random-effect model using the DerSimonian \& Laird method was implemented to bring the individual effect-size estimates together, and the estimate of heterogeneity was taken from the Mantel-Haenszel model (Cohn and Becker, 2003).

The concordance of Ser326Cys genotypes with Hardy-Weinberg proportions was calculated using the $\chi^{2}$ test or Fisher exact test in control groups. Possible heterogeneity between the results of individual studies or in groups defined by race, study design, or genotyping method was assessed using the inconsistency index $I^{2}$ statistic (ranging from 0 to $100 \%$ ) with higher values suggesting the existence of heterogeneity (Higgins and Thompson, 2002; Higgins et al., 2003). In the case of between-study heterogeneity, we examined the study characteristics that could stratify the studies into subgroups with homogeneous effects.

Funnel plots and Egger regression asymmetry tests were used to examine publication bias. Probability less than 0.05 was judged significant except for the $I^{2}$ statistic and, for publication, Egger's statistic, where a significance level of less than 0.1 was chosen. Data management and statistical analyses were performed using STATA version 11.0 for Windows (Wang et al., 2012).

\section{RESULTS}

\section{Single-locus analysis}

The success rates of genotyping the Ser326Cys polymorphism were 97.32 and $100 \%$ in patients and controls, respectively. The genotype distributions of the examined polymorphism followed Hardy-Weinberg equilibrium in controls $(\mathrm{P}>0.05)$. There were no significant differences in the genotype and allele distributions of the Ser326Cys polymorphism between patients with gastric cancer and controls $(\mathrm{P}=0.8026$ for genotype, and $\mathrm{P}=0.5857$ for allele), and this non-significance was also mirrored under assumptions of the additive $(\mathrm{OR}=0.95$; $95 \% \mathrm{CI}=0.78-1.15 ; \mathrm{P}=0.588)$, dominant $(\mathrm{OR}=0.96 ; 95 \% \mathrm{CI}=0.72-1.28 ; \mathrm{P}=0.786)$ and recessive $(\mathrm{OR}=0.88 ; 95 \% \mathrm{CI}=0.61-1.27 ; \mathrm{P}=0.509)$ models $($ Table 1$)$. 
Table 1. Alleles and genotype distributions of the $h O G G 1$ gene Ser326Cys polymorphism between cases $(\mathrm{N}=436)$ and controls $(\mathrm{N}=372)$.

\begin{tabular}{|c|c|c|c|c|c|}
\hline \multirow[t]{2}{*}{ Status } & \multicolumn{3}{|c|}{ Ser326Cys genotypes $(\mathrm{N})$} & \multicolumn{2}{|c|}{ Ser326Cys alleles $(\%)$} \\
\hline & SerSer & SerCys & CysCys & Ser & Cys \\
\hline Cases & 154 & 210 & 72 & 59.40 & 40.60 \\
\hline \multirow[t]{2}{*}{ Controls } & 128 & 176 & 68 & 58.06 & 41.94 \\
\hline & \multicolumn{3}{|c|}{$\chi^{2}=0.4397 ; \mathrm{P}=0.8026$} & \multicolumn{2}{|c|}{$\chi^{2}=0.2972 ; \mathrm{P}=0.5857$} \\
\hline
\end{tabular}

$\begin{array}{ccc}{\text { Additive } \text { model }^{\mathrm{a}}} & \text { Dominant model }^{\mathrm{a}} & \text { Recessive model }^{\mathrm{a}} \\ 0.95 ; 0.78-1.15 ; 0.588 & 0.96 ; 0.72-1.28 ; 0.786 & 0.88 ; 0.61-1.27 ; 0.509\end{array}$

$\mathrm{P}$ values were calculated using the $\chi^{2}$-test from a series of $3 \times 2$ contingency tables for genotype data and $2 \times 2$ contingency tables for allele data. ${ }^{a}$ Data are reported as odds ratio; $95 \%$ confidence interval; $\mathrm{P}$ values for genetic modes of inheritance.

\section{Eligible articles for meta-analysis and study characteristics}

The initial search yielded 25 potentially relevant articles. After applying the inclusion/ exclusion criteria, 13 articles were eligible for inclusion in the meta-analysis (Shinmura et al., 1998; Hanaoka et al., 2001; Takezaki et al., 2002; Tsukino et al., 2004; Poplawski et al., 2006; Capellá et al., 2008; Farinati et al., 2008; Canbay et al., 2010; Malik et al., 2010; Palli et al., 2010; Sun et al., 2010; Liu et al., 2011; Engin et al., 2011). In total, 14 separate studies plus the present study, encompassing a total of 2745 patients with gastric cancer and 4588 controls, were meta-analyzed, with seven studies conducted in Asians, four in Caucasians, and four in other populations.

Besides the present study, twelve studies were conducted using a hospital-based design and three studies used a population-based design. Thirteen studies utilized a PCR-based genotyping method, while two studies utilized TaqMan or probe methodologies. Baseline characteristics of the qualified studies are shown in Table 2. The genotype distributions of the hOGG1 gene Ser326Cys polymorphism were in agreement with Hardy-Weinberg equilibrium among control groups of all studies.

Table 2. Baseline characteristics of all eligible studies.

\begin{tabular}{|c|c|c|c|c|c|c|c|c|c|c|c|}
\hline References & Race & Country & $\begin{array}{l}\text { Sources } \\
\text { of Con. }\end{array}$ & $\begin{array}{l}\text { Genotyping } \\
\text { method }\end{array}$ & $\begin{array}{c}\mathrm{Ca} \\
\text { SerSer }\end{array}$ & $\begin{array}{c}\mathrm{Ca} \\
\text { SerCys }\end{array}$ & $\begin{array}{c}\mathrm{Ca} \\
\text { CysCys }\end{array}$ & $\begin{array}{l}\text { Con } \\
\text { SerSer }\end{array}$ & $\begin{array}{l}\text { Con } \\
\text { SerCys }\end{array}$ & $\begin{array}{c}\text { Con } \\
\text { CysCys }\end{array}$ & HWE \\
\hline Shinmura (1998) & Asian & Japanese & $\mathrm{HCC}$ & PCR-SSCP & 9 & 16 & 10 & 15 & 20 & 7 & $>0.05$ \\
\hline Hanaoka (2001) & Others & Japanese Brazilians & $\mathrm{HCC}$ & PCR-SSCP & 20 & 29 & 9 & 44 & 56 & 27 & $>0.05$ \\
\hline Hanaoka (2001) & Others & non-Japanese Brazilians & $\mathrm{HCC}$ & PCR-SSCP & 133 & 67 & 8 & 123 & 74 & 8 & $>0.05$ \\
\hline Takezaki (2002) & Asian & China & PCC & PCR-SSCP & 20 & 61 & 20 & 30 & 120 & 48 & $>0.05$ \\
\hline Tsukino (2004) & Asian & Japanese & $\mathrm{HCC}$ & PCR-SSCP & 32 & 75 & 35 & 74 & 141 & 56 & $>0.05$ \\
\hline Poplawski (2006) & Caucasian & Poland & $\mathrm{HCC}$ & PCR-SSCP & 22 & 6 & 0 & 18 & 15 & 0 & $>0.05$ \\
\hline Capella (2008) & Caucasian & Spain & PCC & Probe & 279 & 137 & 22 & 621 & 352 & 53 & $>0.05$ \\
\hline Farinati (2008) & Caucasian & Italy & $\mathrm{HCC}$ & PCR-RFLP & 33 & 15 & 2 & 36 & 7 & 0 & $>0.05$ \\
\hline Palli (2010) & Caucasian & Italy & PCC & TaqMan & 192 & 101 & 11 & 325 & 191 & 29 & $>0.05$ \\
\hline Malik (2010) & Asian & India & $\mathrm{HCC}$ & PCR-SSCP & 50 & 51 & 7 & 94 & 89 & 12 & $>0.05$ \\
\hline Sun (2010) & Asian & China & $\mathrm{HCC}$ & PCR-RFLP & 21 & 19 & 33 & 72 & 119 & 64 & $>0.05$ \\
\hline Canbay (2010) & Others & Turkey & $\mathrm{HCC}$ & PCR-RFLP & 24 & 13 & 3 & 171 & 69 & 7 & $>0.05$ \\
\hline Liu (2011) & Asian & China & $\mathrm{HCC}$ & PCR-HMR & 114 & 302 & 202 & 144 & 447 & 322 & $>0.05$ \\
\hline Engin (2011) & Others & Turkey & $\mathrm{HCC}$ & PCR-RFLP & 53 & 42 & 11 & 51 & 47 & 18 & $>0.05$ \\
\hline $\mathrm{Hu}$ (the present study) & Asian & China & $\mathrm{HCC}$ & PCR-LDR & 154 & 210 & 72 & 128 & 176 & 68 & $>0.05$ \\
\hline
\end{tabular}

$\mathrm{HCC}=$ hospital-based case-control study; $\mathrm{PCC}=$ population-based case-control study; $\mathrm{Ca}=$ case; $\mathrm{Con}=$ control; HWE $=$ Hardy-Weinberg equilibrium in the control group; PCR-SSCP $=$ PCR-single strand conformational polymorphism; PCR-RFLP = PCR-restriction fragment length polymorphism; PCR-HMR = PCR-high-resolution melting curve; PCR-LDR = PCR-ligase detection reactions. 


\section{Meta-analysis results}

After combining all studies qualified, we found a null association of the $h O G G 1$ gene Ser326Cys polymorphism with gastric cancer under both allelic $(\mathrm{OR}=1.02 ; 95 \% \mathrm{CI}=0.91$ $1.14 ; \mathrm{P}=0.739)$ and dominant $(\mathrm{OR}=0.97 ; 95 \% \mathrm{CI}=0.78-1.21 ; \mathrm{P}=0.803)$ models, and this association suffered from significant evidence of heterogeneity between studies (allelic and dominant models: $I^{2}=41.7 \%$ and $39.9 \%$ ) (Figure 1). However, there was low probability of publication bias for both models $\left(\mathrm{P}_{\text {Egger }}=0.163\right.$ and 0.404$)$ (Figure 2).
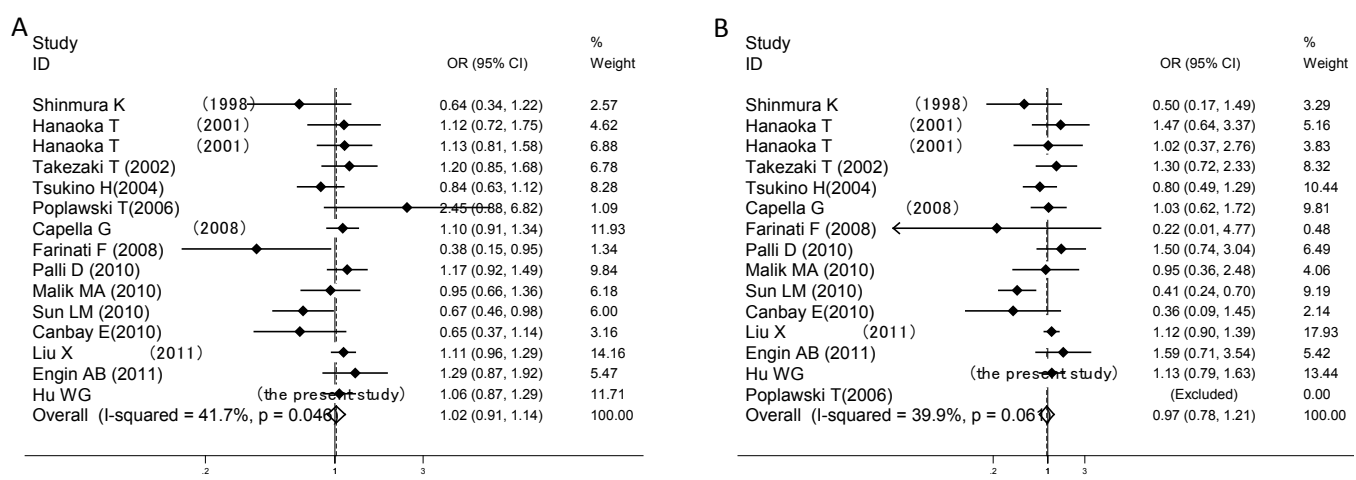

Figure 1. Forest plots of the $h O G G 1$ gene Ser326Cys polymorphism with gastric cancer under both allelic (A) and dominant (B) models.

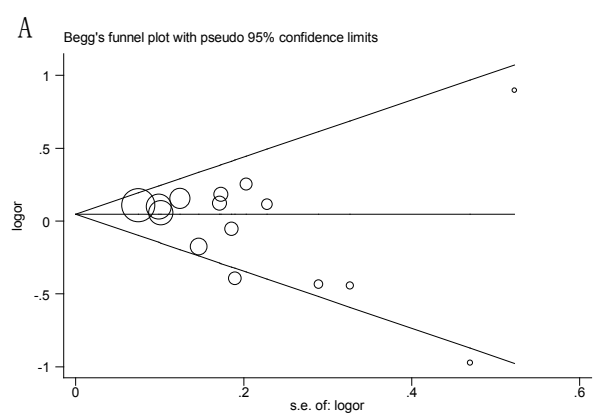

$\mathrm{C}$

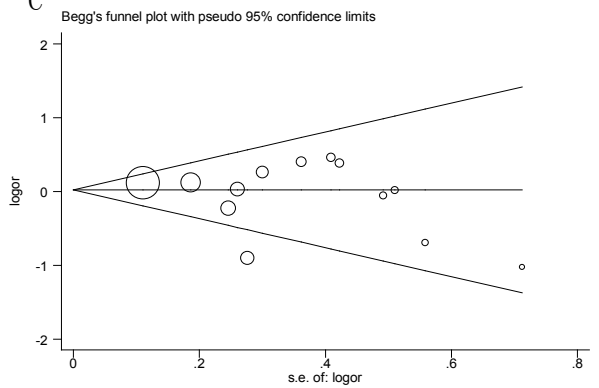

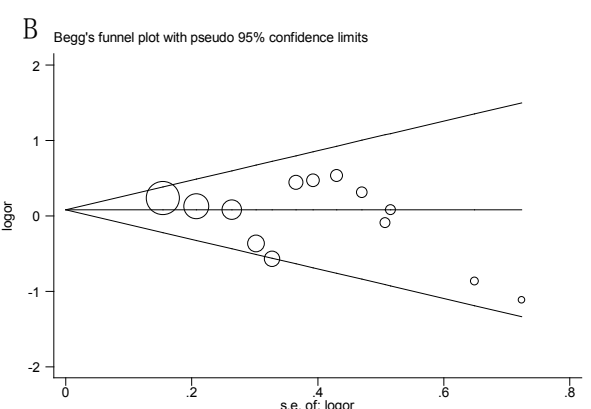

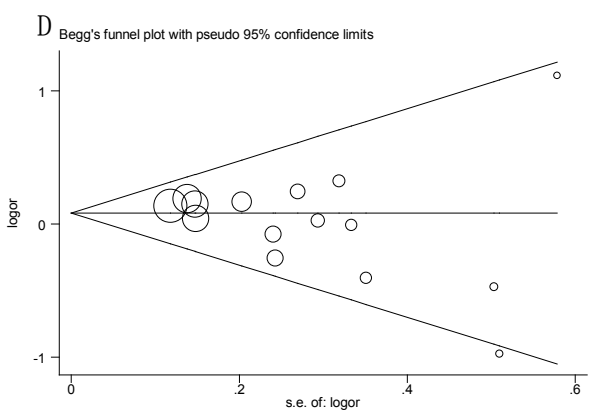

Figure 2. Begg's funnel plots of publication bias tests for the hOGG1 Ser326Cys polymorphism. A. 326Ser allele vs 326Cys allele. B. 326 SerSer vs 326 CysCys. C. Dominant model. D. Recessive model. 
Considering the fact that ethnicity differences might bias the overall estimates, we therefore conducted separate analyses based on subject ethnicity. We classified seven study populations as Asian, 4 as Caucasian, and 4 as "other" population groups. As shown in Table 3 , comparison of $326 \mathrm{Ser}$ versus $326 \mathrm{Cys}$ generated a weakly protective albeit nonsignificant tendency for gastric cancer incidence in Asians $(\mathrm{OR}=0.97 ; 95 \% \mathrm{CI}=0.91-1.14 ; \mathrm{P}=0.495)$, whereas a contrary tendency was observed in Caucasians $(\mathrm{OR}=1.08 ; 95 \% \mathrm{CI}=0.79-1.49$; $\mathrm{P}=0.616)$ and in "Others" $(\mathrm{OR}=1.07 ; 95 \% \mathrm{CI}=0.84-1.37 ; \mathrm{P}=0.574)$. Similar tendencies were noted for the other genetic models except for Asians in the recessive model $(\mathrm{OR}=1.05$; $95 \% \mathrm{CI}=0.90-1.23 ; \mathrm{P}=0.538)($ Table 3$)$.

To account for potential sources of heterogeneity, we also conducted a set of subgroup analyses according to the source of controls and the genotyping method. Upon stratification by control source, no significant association was detected in the comparison between hospitaland population-based groups. However, these two groups exhibited contrary tendencies. Similarly, upon stratification by genotyping method, the PCR-based group and the TaqMan or probe groups also showed contrary tendencies, although no evidence of significance was identified between these two groups.

\section{DISCUSSION}

As hOGG1 has an important role in DNA repair, it is biologically plausible that hOGG1 genetic polymorphism might modulate the risk of various cancers, with respect to the $h O G G 1$ Ser326Cys polymorphism in particular. A meta-analysis of ten case-control studies suggested that the hOGG1 326Cys allele had a significant protective effect for breast cancer in European women (Yuan et al., 2010). Furthermore, a meta-analysis of eight casecontrol studies suggested that the hOGG1 Ser326Cys polymorphism was associated with hepatocellular carcinoma risk among East Asians (Wang et al., 2013).

Although numerous studies have regarded the $h O G G 1$ gene Ser326Cys polymorphism as a promising candidate for gastric cancer, our case-control study in a large Han Chinese population, along with the subsequent meta-analysis, failed to confirm this relationship, even across different ethnic populations. However, we found that there was a low probability of publication bias for all genotypic models, indicating the robustness of our findings. To the authors' knowledge, this is the most comprehensive meta-analysis investigating the genetic susceptibility of $h O G G 1$ gene Ser326Cys polymorphism variants to gastric cancer.

Several strengths distinguishing the present investigation merit consideration. First, this is to date the largest synthesis exploring the association of the hOGG1 gene Ser326Cys polymorphism with gastric cancer. Second, the results of the present case-control study were in line with those of the corresponding meta-analysis. Furthermore, this updated meta-result was similar to those from previous meta-analyses (Wang et al., 2011; Li et al., 2012). Third, our results are little prone to selection bias in view of the low identified probability of publication bias.

In addition, some limitations should be considered when interpreting our findings. First, as with all meta-analyses, publication bias might have occurred because our analyses were based entirely on published studies from English- and Chinese-language journals. Second, although the adopted random-effect model takes both between-study variance and within-study variances into account, this model cannot be regarded as a panacea for heterogeneity (Spector and Thompson, 1991). Furthermore, as stated by Higgins et al. (2009), 


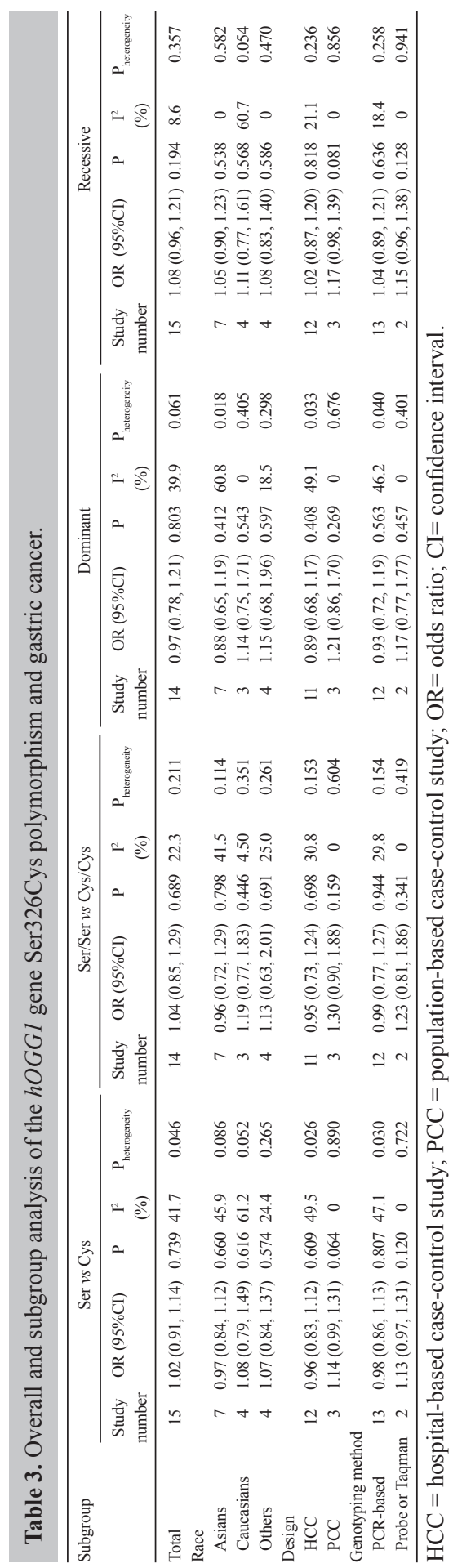


the assumption of true quantities from the individual studies following a certain probability distribution in a random-effect model is somewhat arbitrary and makes the interpretation of its predictions difficult. Third, we focused on only one polymorphism in the hOGG1 gene, and did not cover other susceptibility genes or polymorphisms. Given these limitations, we cannot jump to a final conclusion until further verification of our findings in vitro, in vivo, and in large prospective studies.

In summary, this case-control study in Han Chinese, along with the comprehensive meta-analysis, failed to confirm the association of the $h O G G 1$ gene Ser326Cys polymorphism with gastric cancer risk, even across different ethnic populations. Nevertheless, for practical reasons, we hope that this study will not remain just another endpoint of research instead of a starting point to establish the background data to further investigate the molecular mechanisms of the $h O G G 1$ gene and gastric cancer.

\section{REFERENCES}

Canbay E, Agachan B, Gulluoglu M, Isbir T, et al. (2010). Possible associations of APE1 polymorphism with susceptibility and HOGG1 polymorphism with prognosis in gastric cancer. Anticancer Res. 30: 1359-1364.

Capellá G, Pera G, Sala N, Agudo A, et al. (2008). DNA repair polymorphisms and the risk of stomach adenocarcinoma and severe chronic gastritis in the EPIC-EURGAST study. Int. J. Epidemiol. 37: 1316-1325.

Cohn LD and Becker BJ (2003). How meta-analysis increases statistical power. Psychol. Methods 8: 243-253.

Collins AR and Gaivão I (2007). DNA base excision repair as a biomarker in molecular epidemiology studies. Mol. Aspects Med. 28: 307-322.

Engin AB, Karahalil B, Engin A and Karakaya AE (2011). DNA repair enzyme polymorphisms and oxidative stress in a Turkish population with gastric carcinoma. Mol. Biol. Rep. 38: 5379-5386.

Farinati F, Cardin R, Bortolami M, Nitti D, et al. (2008). Oxidative DNA damage in gastric cancer: CagA status and $O G G 1$ gene polymorphism. Int. J. Cancer 123: 51-55.

Hanaoka T, Sugimura H, Nagura K, Ihara M, et al. (2001). hOGG1 exon7 polymorphism and gastric cancer in case-control studies of Japanese Brazilians and non-Japanese Brazilians. Cancer Lett. 170: 53-61.

Higgins JP and Thompson SG (2002). Quantifying heterogeneity in a meta-analysis. Stat. Med. 21: 1539-1558.

Higgins JP, Thompson SG, Deeks JJ and Altman DG (2003). Measuring inconsistency in meta-analyses. BMJ 327: 557-560.

Higgins JP, Thompson SG and Spiegelhalter DJ (2009). A re-evaluation of random-effects meta-analysis. J. R. Stat. Soc. Ser. A Stat. Soc. 172: 137-159.

Hu WG, Hu JJ, Cai W, Zheng MH, et al. (2014). The NAD(P)H: quinine oxidoreductase 1 (NQO1) gene $609 \mathrm{C}>\mathrm{T}$ polymorphism is associated with gastric cancer risk: evidence from a case-control study and a meta-analysis. Asian Pac. J. Cancer Prev. 15: 2363-2367.

Kohno T, Shinmura K, Tosaka M, Tani M, et al. (1998). Genetic polymorphisms and alternative splicing of the $h O G G 1$ gene, that is involved in the repair of 8-hydroxyguanine in damaged DNA. Oncogene 16: 3219-3225.

Li BR, Zhou GW, Bian Q and Song B (2012). Lack of association between the hOGG1 Ser326Cys polymorphism and gastric cancer risk: a meta-analysis. Asian Pac. J. Cancer Prev. 13: 1145-1149.

Liu X, Xiao N, Guo W, Wu Y, et al. (2011). The $h O G G 1$ gene 5'-UTR variant c.-53G $>$ C contributes to the risk of gastric cancer but not colorectal cancer in the Chinese population: the functional variation of $h O G G 1$ for gastric cancer risk. J. Cancer Res. Clin. Oncol. 137: 1477-1485.

Long N, Moore MA, Chen W, Gao CM, et al. (2010). Cancer epidemiology and control in north-East Asia - past, present and future. Asian Pac. J. Cancer Prev. 11: 107-148.

Malik MA, Zargar SA and Mittal B (2010) Lack of influence of DNA repair gene OGG1 codon 326 polymorphisms of gastric cancer risk in the Kashmir valley. Asian Pac. J. Cancer Prev. 11: 165-168.

Palli D, Polidoro S, D'Errico M, Saieva C, et al. (2010). Polymorphic DNA repair and metabolic genes: a multigenic study on gastric cancer. Mutagenesis 25: 569-575.

Poplawski T, Arabski M, Kozirowska D, Blasinska-Morawiec M, et al. (2006). DNA damage and repair in gastric cancer-a correlation with the $h O G G 1$ and RAD51 genes polymorphisms. Mutat. Res. 601: 83-91.

Ramaniuk VP, Nikitchenko NV, Savina NV, Kuzhir TD, et al. (2014). Polymorphism of DNA repair genes OGG1, XRCC1, XPD and ERCC6 in bladder cancer in Belarus. Biomarkers 19:509-516. 
Shinmura K, Kohno T, Kasai H, Koda K, et al. (1998). Infrequent mutations of the $h O G G 1$ gene, that is involved in the excision of 8-hydroxyguanine in damaged DNA, in human gastric cancer. Jpn. J. Cancer Res. 89: 825-828.

Spector TD and Thompson SG (1991). The potential and limitations of meta-analysis. J. Epidemiol. Community Health 45: 89-92.

Sun LM, Shang Y, Zeng YM, Deng YY, et al. (2010). HOGG1 polymorphism in atrophic gastritis and gastric cancer after Helicobacter pylori eradication. World J. Gastroenterol. 16: 4476-4482.

Takezaki T, Gao CM, Wu JZ, Li ZY, et al. (2002). hOGG1 Ser(326)Cys polymorphism and modification by environmental factors of stomach cancer risk in Chinese. Int. J. Cancer 99: 624-627.

Tsukino H, Hanaoka T, Otani T, Iwasaki M, et al. (2004). hOGG1 Ser326Cys polymorphism, interaction with environmental exposures, and gastric cancer risk in Japanese populations. Cancer Sci. 95: 977-983.

Wang W, Dang S, Li Y, Sun M, et al. (2013). hOGG1 Ser326Cys polymorphism and risk of hepatocellular carcinoma among East Asians: a meta-analysis. PLoS One 8: e60178.

Wang Z, Hu J, Cai W and Zhong J (2011). Lack of association between the 8-oxoguanine DNA glycosylase gene Ser326Cys polymorphism and gastric cancer: evidence from a meta-analysis. Asian Pac. J. Cancer Prev. 12: 3427-3431.

Wang Z, Hu J, Fan R, Zhou J, et al. (2012). Association between CD14 gene C-260T polymorphism and inflammatory bowel disease: a meta-analysis. PLoS One 7: e45144.

Wang ZT, Hu JJ, Fan R, Zhou J, et al. (2014). RAGE gene three polymorphisms with Crohn's disease susceptibility in Chinese Han population. World J. Gastroenterol. 20: 2397-2402.

Yuan W, Xu L, Feng Y, Yang Y, et al. (2010). The hOGG1 Ser326Cys polymorphism and breast cancer risk: a metaanalysis. Breast Cancer Res. Treat. 122: 835-842. 